\title{
Biodiversidade de mosquitos (Diptera: Culicidae) nos parques da cidade de São Paulo I
}

\author{
Antônio Ralph Medeiros-Sousa ${ }^{1}$, Walter Ceretti-Junior ${ }^{1}$, Paulo Roberto Urbinatti', \\ Delsio Natal ${ }^{1}$, Gabriela Cristina de Carvalho ${ }^{1}$, Marcia Bicudo de Paula ${ }^{1}$, Aristides Fernandes ${ }^{\text {, }}$ \\ Maria Helena Silva Homem de Mello², Rosane Correia de Oliveira², Lilian Dias Orico
}

Elisabeth Fernandes Bertoletti Gonçalves ${ }^{3}$ \& Mauro Toledo Marrelli ${ }^{1,4}$

${ }^{1}$ Departamento de Epidemiologia, Faculdade de Saúde Pública, Universidade de São Paulo - USP, CEP 01246-904, São Paulo, SP, Brasil

${ }^{2}$ Centro de Controle de Zoonoses, Coordenação de Vigilância em Saúde - COVISA, Secretaria Municipal da Saúde - SMS, Prefeitura do Município de São Paulo-PMSP, CEP 01221-010, São Paulo, SP, Brasil ${ }^{3}$ Departamento de Parques e Áreas Verdes, Secretaria do Verde e Meio Ambiente - SVMA, Prefeitura do Municipio de São Paulo-PMSP, CEP 04103-000, São Paulo, SP, Brasil

${ }^{4}$ Autor para correspondencia: Mauro Toledo Marrelli,e-mail: mmarelli@usp.br

MEDEIROS-SOUSA, A.R., CERETTI-JUNIOR, W., URBINATTI, P.R., NATAL, D., CARVALHO, G.C., PAULA, M.B., FERNANDES, A., HOMEM DE MELO, M.H.S., OLIVEIRA, R.C., ORICO, L.D., GONÇALVES, E.F.B. \& MARRELLI, M.T. Mosquito (Diptera: Culicidae) survey in parks of São Paulo City I. Biota Neotrop. 13(1): http://www.biotaneotropica.org.br/v13n1/en/abstract?inventory+bn03813012013

\begin{abstract}
Given the scarcity of information on mosquitoes (Diptera: Culicidae) in the city of São Paulo, led the authors to propose a project to investigate this group of insects in some of the green areas, represented by municipal parks. The captures were undertaken in 35 municipal parks in the south, north and central-west of the city, between October 2010 and February 2011, during daylight. Immature forms were collected from breeding places with entomological ladles and suction pumps and adults from resting places with electric aspirators (12V battery). The identification of the specimens was undertaken in the Culicid laboratory of the Faculdade de Saúde Pública, Universidade de São Paulo. In this first phase of the project, a total of 5,129 specimens distributed in 11 genera and 41 taxonomic categories were captured. Culex (Cux) quinquefasciatus was the most abundant species. The genus Aedes was represented mainly by Ae. (Och) fluviatilis and Ae. (Ste) albopictus. Ae. (Ste) aegypti and Ae. (Och) scapularis were frequent in some parks. Other genera were less abundant. Of the parks investigated, 25.7\% presented more than ten groups of species, notably the Anhanguera with 26; on the other hand, $57.1 \%$ had five or fewer groups. Despite the anthropic pressure on these environments, several culicid species make use of these habitats as refuges. It is recommended that these environments be kept under constant epidemiological surveillance as some of the species collected constitute public health threats as pathogen vectors to the human population.
\end{abstract}

Keywords: biodiversity, culicids, mosquitoes, parks.

MEDEIROS-SOUSA, A.R., CERETTI-JUNIOR, W., URBINATTI, P.R., NATAL, D., CARVALHO, G.C., PAULA, M.B., FERNANDES, A., HOMEM DE MELO, M.H.S., OLIVEIRA, R.C., ORICO, L.D., GONÇALVES, E.F.B. \& MARRELLI, M.T. Biodiversidade de mosquitos (Diptera: Culicidae) nos parques da cidade de São Paulo I. Biota Neotrop. 13(1): http://www.biotaneotropica.org.br/v13n1/pt/abstract?inventory+bn03813012013

Resumo: Diante da escassez de informações sobre mosquitos (Diptera: Culicidae) na cidade de São Paulo, foi proposto um projeto para estudar esse grupo de insetos nas áreas verdes representadas pelos parques municipais da cidade. Foram investigados 35 desses parques distribuídos nas regiões sul, norte e centro-oeste da cidade, entre outubro de 2010 e fevereiro de 2011 em período diurno. Os imaturos foram coletados dos criadouros por meio de conchas entomológicas e bomba de sucção e os adultos foram capturados em seus abrigos por aspirador elétrico (bateria de 12V). A identificação e catalogação de espécimes foram feitas no Laboratório de Entomologia da Faculdade de Saúde Pública, Universidade de São Paulo. Nesta primeira fase do projeto, coletou-se um total de 5.129 espécimes distribuídos em 11 gêneros e 41 categorias taxonômicas. Culex (Cux.) quinquefasciatus foi a espécie mais abundante. O gênero Aedes foi representado principalmente por Ae. (Och.) fluviatilis e Ae. (Ste.) albopictus. Ae. (Ste.) aegypti e Ae. (Och.)scapularis também foram frequentes em alguns parques. Os demais gêneros apresentaram-se pouco abundantes. Dos parques, 25,7\% apresentaram mais de dez grupos, com destaque para o Anhanguera com 26; em contrapartida, 57,1\% apresentaram cinco ou menos grupos. Apesar da pressão antrópica sobre esses ambientes, diversas espécies de culicídeos se utilizam destes habitats para a manutenção e refúgio de suas populações. É recomendado que estes ambientes estejam sob constante vigilância epidemiológica, visto que algumas das espécies coletadas possuem importância em saúde pública como vetoras de patógenos à população humana.

Palavras-chave: biodiversidade, culicideos, mosquitos, parques. 


\section{Introdução}

Os mosquitos (Diptera: Culicidae) constituem grupo taxonômico de relevante biodiversidade com cerca de 3.500 espécies descritas (Stone et al. 1959, Belkin et al. 1965, Guimarães 1997, Harbach 2011), adaptadas aos mais diversos ambientes, das florestas às áreas urbanizadas, muitas delas, desempenham atividade vetora na transmissão de agentes patogênicos ao homem e a outros vertebrados (Consoli \& Lourenço-de-Oliveira 1994, Forattini 2002).

Nos últimos 50 anos, as intensas modificações ocorridas no Brasil incluem a migração da população humana do ambiente rural para aglomerados urbanos. Consequentemente alterou-se também o espectro das doenças, com redução das endemias rurais e aumento da frequência de agravos das sociedades modernas como causa de morbi-mortalidade (Chaimowicz 1997). Contudo, doenças cujos agentes etiológicos são veiculados por vetores biológicos continuam sendo problemas em saúde pública em áreas urbanas. Espécies exóticas como Aedes (Stegomyia) aegypti (Linnaeus 1762), Ae. (Ste.) albopictus (Skuse 1894) e Culex (Culex) quinquefasciatus Say 1823 devido às suas implicações epidemiológicas tem sido amplamente estudadas no Brasil. Entretanto, pouco se conhece a respeito dos culicídeos urbanos autóctones, possivelmente porque as espécies nativas registradas nas cidades não têm sido incriminadas como transmissoras de patógenos à população. Tal condição não exclui a possibilidade dessas espécies apresentarem competência e capacidade vetorial para disseminar agentes patogênicos.

A cidade de São Paulo tem atualmente população estimada em 11,2 milhões de habitantes. Ao se considerar os municípios que compõem a Grande São Paulo, sua população alcança a cifra de 20,5 milhões de habitantes (Instituto... 2010) sofrendo, portanto, o estresse ambiental próprio das maiores metrópoles do planeta. A fauna de culicídeos dessa cidade é pouco conhecida. Trabalhos pontuais, em áreas periféricas, indicam abundância relativamente elevada de mosquitos (Urbinatti et al. 2001, Taipe-Lagos \& Natal 2003, Montes 2005, Laporta et al. 2006, Silvério \& Urbinatti 2011). Esses registros sugerem que alguns membros desse táxon subsistiram ou adaptaramse ao processo de urbanização nesta metrópole.

Em São Paulo há parques municipais que preservam resquícios da vegetação original, comportam lagos, servem de abrigo para aves e mamíferos e são visitados continuamente pela população como ambientes de lazer. A prefeitura dessa cidade planeja até o final da atual administração, atingir 100 parques municipais em atividade. Essas "ilhas verdes" têm condições de manter populações de algumas espécies de mosquitos ou servir de refúgio para espécies exóticas que infestam as áreas densamente habitadas da malha urbana como $A e$. aegypti, Ae. albopictus e Cx. quinquefasciatus.

Devido à pressão cada vez mais intensa do homem sobre os ambientes naturais há um crescente risco de contato com agentes patogênicos provenientes desses meios. As arboviroses estariam entre os agravos que mais ameaçam as áreas urbanas, como ocorrido no passado com a febre amarela urbana e com a dengue no presente. Na capital Paulista, o arbovírus Ilhéus, com potencial de infectar humanos, foi isolado em área densamente habitada, representada pelo Parque Ecológico do Tietê (Pereira et al. 2001). Ressalta-se que a cidade de São Paulo está inserida na área da Mata Atlântica do Brasil, região incluída entre os 25 "hotspots" do planeta, locais que devem ser prioritariamente investigados (Myers et al. 2000).

Diante do exposto, justifica-se a realização de estudo da fauna de mosquitos associada às áreas protegidas utilizadas para lazer no interior da malha urbana da Cidade, bem como investigar aspectos ecológicos e epidemiológicos dessas populações. Para esse fim desenvolveu-se um projeto de pesquisa envolvendo as equipes: de entomologia do Departamento de Epidemiologia da Faculdade de Saúde Pública da Universidade de São Paulo (FSP/USP), do Laboratório de Fauna Sinantrópica do Centro de Controle de Zoonoses da Prefeitura Municipal de São Paulo (Labfauna/CCZ), do Departamento de Parques e Áreas Verdes-3, da Secretaria municipal do Verde e do Meio Ambiente de São Paulo (DEPAVE-3/SVMA) e do Departamento de Protozoologia do Instituto de Medicina Tropical da USP (IMT/USP). Em uma primeira etapa do estudo realizou-se um levantamento de espécies de culicídeos em 35 dos principais parques municipais, cujos resultados são apresentados no presente artigo.

\section{Material e Métodos}

Coletas. Culicídeos adultos e imaturos foram coletados entre outubro de 2010 e fevereiro de 2011 em 35 parques municipais, assim distribuídos, segundo as regiões: sul (18), norte (9) e centrooeste (8). Cada parque foi visitado uma vez pela equipe, sempre no período diurno.

As formas adultas foram coletadas por aspiração mecânica durante 20 minutos utilizando-se um aspirador a bateria de 12V (Natal $\&$ Marucci 1984). Foram feitas de uma a três aspirações por parque, dependendo das dimensões e características ambientais do local. As formas imaturas foram coletadas utilizando concha entomológica e sucção com pipeta em diferentes criadouros.

Identificação dos espécimes. Os Culicídeos obtidos nas coletas foram acondicionados adequadamente e transportados para o laboratório de Entomologia (LESP) da Faculdade de Saúde Pública/ Universidade de São Paulo. Neste, os imaturos foram mantidos em desenvolvimento até a fase adulta. A identificação morfológica baseou-se em Lane (1953), Consoli \& Lourenço-de-Oliveira (1994) e Forattini (2002) e as abreviações dos nomes seguiram Reinert (2001).

\section{Resultados}

No período estudado foi obtido um total de 5.129 exemplares, sendo 2.923 mosquitos adultos e 2.206 formas imaturas, totalizando 41 categorias taxonômicas distribuídas em 11 gêneros, conforme apresentado na Tabela 1.

O gênero Culex Linnaeus 1758, presente em todos os parques, foi o que mais contribuiu para o total de categorias taxonômicas, apresentando também as espécies com as maiores frequências: $C x$. quinquefasciatus com 1.040 exemplares (20,28\%) coletados em 27 parques, Cx. (Cux.) spp. com 922 exemplares (17,98\%) coletados em 22 parques e Cx. (Cux.) declarator Dyar \& Knab 1906, 808 exemplares $(15,75 \%)$ coletados em 10 parques.

Aedes esteve presente, principalmente, com as espécies Ae. (Ochlerotatus.) fluviatilis Lutz 1904 (8,09\%) e Ae. albopictus (5,85\%). Ressalta-se a ocorrência de Ae. aegypti, com 1,95\% do total coletado, sendo 4 alados e 96 imaturos em sete dos parques pesquisados; no parque Ibirapuera coletou-se o maior número de espécimes (53 imaturos e três adultos,um macho e duas fêmeas).

Os demais gêneros apresentaram baixa freqüência $(<1 \%)$. Quatro espécies de Anopheles Meigen 1818 foram identificadas: An. (Anopheles) fluminensis Root 1927, An. (Nyssorhynchus) strodei Root 1926, An. (Nys.) evansae (Brèthes 1926) e An. (Nys.) albitarsis Lynch Arribalzaga 1878. Os demais gêneros representados foram: Toxorhynchites Theobald ( 3 espécies), Psorophora Robineu-Desvoidy (2), Mansonia Blanchard (2), Limatus Theobald 1901, Uranotaenia Lynch Arribalzaga, Wyeomyia Theobald, Trichoprosopon Theobald 1901e Lutzia Theobald 1903 (um táxon cada).

Dentre os parques que apresentaram maior riqueza destaca-se o Anhanguera com 26 categorias taxonômicas, seguido de Castelo, Ibirapuera e Shangrilá com 16, 15 e 14 respectivamente. 25,7\% dos parques apresentaram mais de dez grupos; em contrapartida, $57,1 \%$ apresentaram cinco ou menos grupos. A maior quantidade 
Tabela 1. Distribuição de categorias taxonômicas de culicídeos adultos e imaturos, coletados em 35 parques municipais de São Paulo, no período de outubro de 2010 a fevereiro de 2011.

Table 1. Distribution of taxonomic categories of adult and immature culicids collected in 35 municipal parks in São Paulo, between October 2010 and February 2011.

\begin{tabular}{|c|c|c|c|c|c|}
\hline Categoria Taxonômica & Parques & Adultos & Imaturos & Total & $\%$ \\
\hline Cx. (Cux.) quinquefasciatus & $\begin{array}{l}\text { BM, SG, NB, LP, GU, SJ, BA, CS, GN, SH, MM, IB, RG, } \\
\text { DO, JF, RM, AN, PV, JA, VB, RA, RT, SF, EC, BU, LZ e NJ }\end{array}$ & 399 & 641 & 1040 & 20,28 \\
\hline Cx. (Cux.) spp. & $\begin{array}{l}\text { BM, SG, CO, NB, GU, JH, SJ, CS, GN, SH, SD, MM, IB, } \\
\text { RG, JF, RM, AN, PV, RT, SV, LZ e CT }\end{array}$ & 900 & 22 & 922 & 17,98 \\
\hline Cx. (Cux.) declarator & GU, JH, SJ, GN, SH, SD, MM, IB, RM e AN & 804 & 4 & 808 & 15,75 \\
\hline Ae. (Och.) fluviatilis & $\begin{array}{l}\text { BM, SG, CO, NB, LP, GU, GN, SH, SD, MM, IB, RG, JF, } \\
\text { PV, CP, RT, SF, EC, AN, SV e NJ }\end{array}$ & 121 & 294 & 415 & 8,09 \\
\hline Cx. (Cux.) bidens & SJ, CS, NJ, GN, CT, NB, SH, MM, IB, RM, AN, SF e LZ & 245 & 71 & 316 & 6,16 \\
\hline Ae. (Ste.) albopictus & $\begin{array}{l}\text { NB, SJ, CS, GN, SH, SD, MM, IB, VB, PV, CP, RT, SF, } \\
\text { EC, AN, SV e NJ }\end{array}$ & 36 & 264 & 300 & 5,85 \\
\hline Cx. (Cux.) dolosus & $\mathrm{BM}, \mathrm{CS}$ e CT & & 296 & 296 & 5,77 \\
\hline Ae. (Och.) scapularis & GU, SJ, CS, NJ, GN, SH, SD, IB, AN, SV e LF & 141 & 5 & 146 & 2,85 \\
\hline Cx. (Cux.) nigripalpus & SG, GU, SJ, CS, NJ, GN, SH, SD, MM, IB, RG, NA e SV & 137 & 5 & 142 & 2,77 \\
\hline$C x .(C u x$.$) grupo Coronator$ & IB, AN, CS, NJ, GN, SH, MM, SV e LF & 15 & 125 & 140 & 2,73 \\
\hline Cx. (Cux.) chidesteri & NB, LP, JH, SJ, CS, GN, SH, SD, MM, IB, CT e JC & 58 & 73 & 131 & 2,55 \\
\hline Ae. (Ste.) aegypti & IB, JF, SG, SD, RG, NA e LZ & 4 & 96 & 100 & 1,95 \\
\hline Cx. (Cux.) brami & $\mathrm{AN}, \mathrm{CP}$ e RA & & 100 & 100 & 1,95 \\
\hline Cx. (Cux.) eduardoi & $\mathrm{JH}, \mathrm{CS}, \mathrm{MM}$ e AN & & 47 & 47 & 0,92 \\
\hline$C x .(M c x$.) grupo Imitator & $\mathrm{SJ}, \mathrm{BA}, \mathrm{SD}$ e SF & 6 & 32 & 38 & 0,74 \\
\hline Cx. (Cux.) coronator & SG, NJ, IB, NA e LZ & 3 & 16 & 19 & 0,37 \\
\hline$T x$. spp. & $\mathrm{NB}, \mathrm{GU}, \mathrm{SD}, \mathrm{IB}, \mathrm{e} \mathrm{SF}$ & & 19 & 19 & 0,37 \\
\hline An. (Ano.) fluminensis & $\mathrm{AN}$ & & 16 & 16 & 0,31 \\
\hline Cx. (Mel.) maxinocca (próximo) & $\mathrm{AN}$ e SV & & 15 & 15 & 0,29 \\
\hline Li. durhami & $\mathrm{JH}, \mathrm{SH}, \mathrm{MM}, \mathrm{AN}$ e BM & 7 & 7 & 14 & 0,27 \\
\hline Ps. (Jan.) ferox & AN & 11 & & 11 & 0,21 \\
\hline Cx. (Cux.) dolosus/eduardoi & BM, SJ, SH, IB, SF e AN & 11 & & 11 & 0,21 \\
\hline Wy. (Pho.) galvaoi & GU e SD & & 11 & 11 & 0,21 \\
\hline An. (Nys.) strodei & $\mathrm{CS}, \mathrm{AN}$ e $\mathrm{GN}$ & 2 & 8 & 10 & 0,19 \\
\hline Cx. (Cux.) lygrus & $\mathrm{NB}, \mathrm{SH}, \mathrm{SD}, \mathrm{AN}, \mathrm{SV}$ e SJ & 8 & 1 & 9 & 0,18 \\
\hline Ur. Nataliae & $\mathrm{JC}$ e CT & & 8 & 8 & 0,16 \\
\hline $\begin{array}{l}\text { Cx. (Mel.) seção Melanoconion } \\
\text { spp. }\end{array}$ & $\mathrm{BA}, \mathrm{AN}$ e $\mathrm{CS}$ & 2 & 5 & 7 & 0,14 \\
\hline Tr. Pallidiventer & SF e SV & & 7 & 7 & 0,14 \\
\hline An. (Nys.) evansae & $\mathrm{AN}, \mathrm{CS}$ e $\mathrm{SV}$ & 1 & 5 & 6 & 0,12 \\
\hline Ma. (Man.) titillans & CS e MM & 6 & & 6 & 0,12 \\
\hline Cx. (Mel.) vaxus & SV & & 4 & 4 & 0,08 \\
\hline Cx. (Mel.) inadimirabilis & $\mathrm{LF}$ & & 2 & 2 & 0,04 \\
\hline Cx. (Phe.) corniger & $\mathrm{SH}$ e MM & 1 & 1 & 2 & 0,04 \\
\hline Lu. bigoti & $\mathrm{AN}$ & & 2 & 2 & 0,04 \\
\hline Tx. haemorrhoidalis & SG & & 2 & 2 & 0,04 \\
\hline An. (Nys.) albitarsis & $\mathrm{AN}$ & & 1 & 1 & 0,02 \\
\hline Cx. (Mcx.) imitator imitator & SD & 1 & & 1 & 0,02 \\
\hline Cx. (Mel.) intrincatus & AN & & 1 & 1 & 0,02 \\
\hline Cx. (Mel.) seção Spisseps sp. & AN & 1 & & 1 & 0,02 \\
\hline Ma. (Man.) humeralis & $\mathrm{CS}$ & 1 & & 1 & 0,02 \\
\hline Ps. Discrucians & $\mathrm{CS}$ & 1 & & 1 & 0,02 \\
\hline Tx. bambusicolus (próximo) & IB & 1 & & 1 & 0,02 \\
\hline Total & & 2923 & 2206 & 5129 & 100,00 \\
\hline
\end{tabular}

Anhanguera (AN), Barragem (BA), Burle Marx (BM), Buenos Aires (BU), Castelo (CS), Cordeiro (CO), Luis Carlos Prestes (CP), Cidade Toronto (CT), São Domingos (DO), Eucaliptos (EC), Guanhembú (GN), Guarapiranga (GU), Ibirapuera (IB), Jacinto Alberto (JA), Jacques Cousteau (JC), Jardim Felicidade (JF), Jardim Herculano (JH), Linear do Fogo (LF), Lina e Paula Raia (LP), Luz (LZ), M'boi Mirim (MM), Nabuco (NB), Nove de Julho (NJ), Previdência (PV), Cohab Raposo Tavares (RA), Rodrigo de Gasperi (RG), Vila dos Remédios (RM), Raposo Tavares (RT), Santo Dias (SD), Colina de São Francisco (SF), Severo Gomes (SG), Shangrilá (SH), São José (SJ), Senhor do Vale (SV), Orlando Villas Boas (VB), \% (abundância relativa). 
de mosquitos adultos foi coletada no parque Guanhembú, com 790 espécimes representados principalmente por Cx. (Cux.) spp. (350) e Cx. declarator (298). O parque Ibirapuera apresentou o maior número de imaturos, com 536 exemplares, predominando Cx. quinquefasciatus (193 espécimes), Ae. albopictus (144) e Ae. fluviatilis (139).

\section{Discussão}

Levantamentos anteriores em diferentes pontos da cidade de São Paulo já registram a presença de táxons em comum com o presente trabalho, tais como: Ae. scapularis, Ae. albopictus, $C x$. (Cux.) chidesteri Dyar 1921, Cx. declarator, Cx. (Cux.) nigripalpus Theobald 1901, dentre outros (Forattini et al. 1973, Urbinatti et al. 2001, Taipe-Lagos \& Natal 2003, Montes 2005, Laporta et al. 2006, Silvério \& Urbinatti 2011).

Com exceção do trabalho de Montes (2005) no Parque Ecológico da Cantareira, os demais mostram marcante presença de $C x$. quinquefasciatus, espécie normalmente associada a coleções de água estagnada com forte carga orgânica. O papel desta espécie como vetor primário da filariose bancroftiana em algumas regiões do Brasil, aliado ao seu potencial para veiculação de arbovírus e incomodo causado à população humana, em razão do seu comportamento antropofílico e de seus altos índices de abundância (Consoli \& Lourenço-de-Oliveira 1994, Forattini 2002, Natal \& Ueno 2004) fazem dela uma das mais importantes do ponto de vista epidemiológico nas cidades. Ressalta-se a ampla presença desta nos parques municipais pesquisados.

O mosquito Aedes aegypti, importante vetor do dengue e febre amarela urbana no Brasil, esteve ausente nos estudos anteriores em outros parques paulistanos. Apesar de esta espécie possuir uma forte associação com o ambiente domiciliar, sua presença em sete parques indica que ambientes deste tipo, totalmente inseridos na mancha urbana, não estão livres da presença deste vetor, associado provavelmente às áreas com maior concentração de funcionários e visitantes. Por outro lado, a espécie Ae. fluviatilis foi o mosquito mais frequente do gênero. Em laboratório, esta espécie é capaz de infectar-se com alguns agentes patogênicos, dentre eles o vírus da febre amarela (Consoli \& Lourenço-de-Oliveira 1994). Também em ensaios laboratoriais, Ae. albopictus, mostrou-se capaz de transmitir pelo menos 22 arbovirus, incluindo febre amarela e dengue, sendo vetor natural desta na Ásia (Gratz 2004). O aparecimento dessas espécies em vários parques pesquisados e em diferentes regiões da cidade ressalta a ampla distribuição e adaptabilidade destas aos mais variados ambientes antropizados.

Dentre os anofelinos encontrados, Na. albitarsis é o que possui maior importância epidemiológica. Este mosquito, amplamente distribuído no Brasil, é considerado vetor secundário do plasmódio da malária humana, sendo, porém, encarado como único transmissor em algumas localidades do País (Consoli \& Lourenço-de-Oliveira 1994). As outras espécies do gênero amostradas na pesquisa possuem menor importância na epidemiologia da malária, mas podem ser consideradas vetoras secundárias. Trabalhos anteriores dentro da cidade não constataram a presença de An. fluminensis. Imaturos desta espécie foram encontrados no parque Anhanguera.

Outras espécies de importância em saúde pública como $C x$. nigripalpus e Ae. (Och.) scapularis (Rondani 1848), incriminadas na transmissão de arbovirus (Forattini et al. 1995, Rey et al. 2006), mesmo apresentando abundância relativa moderada (Tabela 1), mostram-se bem distribuídas entre parques de diferentes regiões. Alguns dos culicídeos coletados no presente trabalho apresentam comportamento predatório em sua fase larval, este é o caso de espécies do gênero Toxorhynchites. As formas adultas deste gênero não possuem hábitos hematófagos, sendo, portanto desprovidas de importância epidemiológica. Devido estas características suas formas imaturas vêm sendo estudadas para aplicação no controle biológico de espécies como Ae. aegypti e Cx. quinquefasciatus. Imaturos de Lutzia bigoti Bellardi 1862 também apresentam comportamento predatório e, assim como os Toxorhynchites, também controlam a população larval de outros culicídeos em seus criadouros. Sabetíneos como Limatus durhami Theobald 1901 são considerados predadores não obrigatórios, isto é, na ausência de alimento ou encontro casual com a presa. Apesar da atividade predatória que exercem em seus criadouros, fêmeas adultas de Lz. bigoti e Li. durhami são hematófagas e, portanto, não são espécies consideradas para estudos de aplicação em controle biológico (Lopes 1999, Forattini 2002).

Diante da pressão antrópica sobre os ambientes pesquisados, nota-se a presença de diversas espécies de culicídeos que se utilizam desses habitats como refúgios para manutenção de suas populações. Keesing et al. (2010) apontam que locais com perda de biodiversidade podem afetar a dinâmica de transmissão de doenças infecciosas. A manutenção e ampliação dessas ilhas verdes inseridas na mancha urbana se fazem necessárias à melhoria da qualidade de vida da população paulistana e preservação da biodiversidade de espécies, inclusive as de mosquitos nativos. Inserida em um ecossistema natural, determinada população de mosquito mantémse controlada por diversas pressões seletivas. Uma vez alterado seu ambiente esta pode desaparecer ou beneficiar-se das novas condições a que esta submetida e tornar-se mais abundante na ausência de fatores limitantes (ex. predadores e competição), passando a atuar como fator de incomodo à população humana, ou ainda, tornar-se competente para a transmissão de patógenos. Observada esta última possibilidade e considerado o estresse ambiental característico de metrópoles como São Paulo, reforça-se a importância da aplicação de programas voltados à vigilância: de vetores, de arbovírus e de animais vertebrados reservatórios.

Estudos sistemáticos de diversidade e arbovírus associados aos mosquitos serão desenvolvidos em parques selecionados nas próximas etapas do projeto, gerando informações que contribuam para o conhecimento sobre aspectos ecológicos, genéticos e epidemiológicos de culicídeos que habitam as "manchas verdes" da cidade de São Paulo.

\section{Agradecimentos}

À Fundação de Amparo à Pesquisa do Estado de São Paulo (Projeto BIOTA 2010/51230-8) pelo financiamento e a todos os membros do Departamento de Epidemiologia da Faculdade de Saúde Pública da Universidade de São Paulo, do Departamento de Parques e Áreas Verdes-3, da Secretaria municipal do Verde e do Meio Ambiente de São Paulo e do Centro de Controle de Zoonoses da Prefeitura Municipal de São Paulo, que contribuíram nas coletas e identificação dos espécimes.

\section{Referências Bibliográficas}

BELKIN, J.N., SCHICK, R.X. \& HEINEMANN, S.J. 1965. Mosquito studies (Diptera: Culicidae). V. Mosquitoes originally described from Middle America. Cont. Am. Entomol. Inst. 1:1-95.

CHAIMOWICZ, F. 1997. Health of Brazilian elderly just before of the $21^{\text {st }}$ century: current problems, forecasts and alternatives. Rev. Saúde Pública 31:184-200. PMid:9497568.

CONSOLI, R.A.G.B. \& LOURENÇO-DE-OLIVEIRA, R. 1994. Principais mosquitos de importância sanitária do Brasil. Editora FIOCRUZ, Rio de Janeiro.

FORATTINI, O.P., ISHIATA, G.K., RABELLO, E.X. \& COTRIM, M.D. 1973. Observações sobre os mosquitos Culex da cidade de São Paulo, Brasil. Rev. Saúde Pública 7:315-30. http://dx.doi.org/10.1590/ S0034-89101973000400001 
FORATTINI, O.P., KAKITANI, I., MASSAD, E. \& MARUCCI, D. 1995. Studies on mosquitoes (Diptera: Culicidae) and anthropic environment: 9-Synanthropy and epidemiological vector role of Aedes scapularis in South-Eastern Brazil. Rev. Saúde Pública 29:199-207. http:// dx.doi.org/10.1590/S0034-89101995000300007

FORATTINI, O.P. 2002. Culicidologia Médica. EdUSP, São Paulo, v.2, 860p.

GRATZ, N.G. 2004. Critical review of the vector status of Aedes albopictus. Med. Vet. Entomol. 18:215-227. http://dx.doi.org/10.1111/j.0269283X.2004.00513.X

GUIMARÃES, J.H. 1997. Systematic database of Diptera of the America South of the United States, family Culicidae. Plêiade/Fapesp, São Paulo, 286p.

HARBACH, RE. 2011. Mosquito Taxonomic Inventory. http://mosquitotaxonomic-inventory.info/ family-culicidae-meigen-1818 (último acesso em 16/02/2012).

INSTITUTO BRASILEIRO DE GEOGRAFIAEESTATÍSTICA-IBGE. 2010. Cidades@São Paulo. http://www.ibge.gov.br/cidadesat/link.php?uf=sp (último acesso em 25/11/2011).

KEESING, F., BELDEN, L.K., DASZAK, P., DOBSON, A., HARVELL, C.D., HOLT, R.D., HUDSON, P., JOLLES, A., JONES, K.E., MITCHELL, C.E., MYERS, S.S., BOGICH, T. \& OSTFELD, R.S. 2010. Impacts of biodiversity on the emergence and transmission of infectious diseases. Nature 468:647-652. http://dx.doi.org/10.1038/nature09575

LANE, J. 1953. Neotropical Culicidae. Ed. Universidade de São Paulo, São Paulo, v.2.

LAPORTA, G.Z., URBINATTI, P.R. \& NATAL, D. 2006. Aspectos ecológicos da população de Culex quinquefasciatus Say (Diptera, Culicidae) em abrigos situados no Parque Ecológico do Tietê, São Paulo, SP. Rev. Bras. Entomol. 50:125-127. http://dx.doi.org/10.1590/S008556262006000100019

LOPES, J. 1999. Ecologia de mosquitos (Diptera, Culicidae) em criadouros naturais e artificiais de área rural do norte do Paraná, Brasil. VIII. Influência das larvas predadoras (Toxorhynchites sp., Limatus durhamii e Culex bigoti sobre a população de larvas de Culex quinquefasciatus e Culex eduardoi. Rev. Bras. Zool. 16:821-826. http://dx.doi.org/10.1590/ S0101-81751999000300018
MONTES, J. 2005. Fauna de Culicidae da Serra da Cantareira, São Paulo, Brasil. Rev. Saúde Pública 39:578-84. http://dx.doi.org/10.1590/S003489102005000400010

MYERS, N., MITTERMEIER, R.A., MITTERMEIER, C.G., FONSECA, G.A.B. \& KENT, J. 2000. Biodiversity hotspots for conservation priorities. Nature 403:853-58. http://dx.doi.org/10.1038/35002501

NATAL, D. \& MARUCCI, D. 1984. Aparelho de sucção tipo aspirador para captura de mosquitos. Rev. Saúde Pública 18:418-420. http://dx.doi. org/10.1590/S0034-89101984000500013

NATAL, D. \& UENO, H.M. 2004. Vírus do Nilo Ocidental: características da transmissão e implicações vetoras. Entomol. Vect. 11:417-33.

PEREIRA, L.E., SUSUKI, A., COIMBRA, T.L.M., SOUZA, R.P. \& CHAMELET, E.L.B. 2001. Arbovírus Ilheus em aves silvestres (Sporophila caerulescense e Molothrus bonariensis). Rev. Saúde Pública 35:119-123. http://dx.doi.org/10.1590/S0034-89102001000200003

REINERT, JF. 2001. Revised list of abbreviatios for genera and subgenera of Culicidae (Diptera) and notes on generic and subgeneric changes. J. Am. Mosq. Control. Assoc. 17:51-55.

REY, J.R., NISHIMURA, N., WAGNER, B., BRAKS, M.A.H., O'CONNELL, S.M. \& LOUNIBOS, P.L. 2006. Habitat segregation of mosquito arbovirus vector en South Florida. J. Med. Entomol. 43:1134-1141. http://dx.doi.org/10.1603/0022-2585(2006)43[1134:HSOMAV]2.0.CO;2

SILVÉRIO, E.C. \& URBINATTI, P.R. 2011. Presença de mosquitos (Diptera: Culicidae) em piscinões na zona leste de São Paulo. Rev. Soc. Bras. Med. Trop. 44:504-507. http://dx.doi.org/10.1590/S0037-86822011000400020

STONE, A., KNIGHT, K.L. \& STARCKE, H. 1959. A synoptic catalog of the mosquitoes of the world (Diptera: Culicidae). The Thomas Say Foundation, Entomol. Soc. Amer. 6:1-358.

TAIPE-LAGOS, C.B. \& NATAL, D. 2003. Abundância de culicídeos em área metropolitana preservada e suas implicações epidemiológicas. Rev. Saúde Pública 37:275-279.

URBINATTI, P.R., SENDACZ, S. \& NATAL, D. 2001. Imaturos de mosquitos (Diptera: Culicidae) em parque de área metropolitana aberto à visitação pública. Rev. Saúde Pública 35:461-466. http://dx.doi.org/10.1590/ S0034-89102001000500009 